\title{
STABILITY ANALYSIS OF SHIPS' MOVEMENT ALONG OPTIMAL ROUTES
}

\author{
IRINA V. ALEKSANDROVA \& ALEXEY P. ZHABKO \\ St. Petersburg State University, Russian Federation
}

\begin{abstract}
In this report, we consider the system of difference-differential equations of neutral type with homogeneous, of the larger unit order, right-hand sides. The following fact is well known. If a system of retarded-type difference-differential equations with homogeneous, of the larger unit order, righthand sides is asymptotically Lyapunov stable at zero delays, then the zero solution of the initial system is also asymptotically Lyapunov stable for any continuous and bounded delays. For this case, the Lyapunov-Krasovskii functional is constructed to estimate the asymptotic stability domain of the zero solution. For a linear system of neutral type, the concept of the Lyapunov matrix is introduced and the Lyapunov-Krasovskii functional is constructed. This functional was then used to analyze exponential stability. This paper presents sufficient conditions for asymptotic Lyapunov stability and Lyapunov instability of the zero solution for a class of homogeneous difference-differential systems of neutral type. In addition, a constructive algorithm for checking the stability and instability of the zero solution is formulated. Another result is the development of a method for constructing a complete type Lyapunov-Krasovskii functional, previously used for the analysis of homogeneous differencedifferential systems of retarded type.

Keywords: time delay systems, neutral type, asymptotic stability, homogeneous systems.
\end{abstract}

\section{INTRODUCTION}

The account of delay in thrusters is necessary for safety of maneuvering of sea vessels in heavy conditions of navigation (mooring, passing narrowness, etc.) [1], [2]. The movement of the sea vessel along the program trajectory leads to a number of problems associated with the implementation of this trajectory. The first problem is the following. If the mathematical model of the ship dynamics is described by a system of ordinary differential equations, then under optimal control the closed system transforms into a system of difference-differential equations of neutral type. Possible approaches to the solution of this problem are either the introduction of an asymptotic observer [3] or compensation for the resulting time-delay [4]. However, such approaches lead to an increase in the dimension of the closed system and complication of control algorithms. Specific examples of accounting for delays in marine facility management systems are presented in detail in the papers [1], [2].

The second problem is related to the nonlinearity of the system in deviations from the program motion. If the first, in a broad sense, approximation of the system [5] does not have a linear component, then it is impossible to apply the known stability theorems by linear approximation. Then we can assume that the right parts of the system of the first, in a broad sense, approximations are described by homogeneous phase variable functions.

In this article, we use the methodology of Lyapunov-Krasovskii functional to analyze the stability of a class of homogeneous differential-difference systems of neutral type. It is proved that if the corresponding homogeneous system with zero delays is asymptotically stable, then the trivial solution of a homogeneous retarded type system is also asymptotically stable for any limited delays [6], [7]. Basing on these results and results [8]-[11], we constructed the complete type Lyapunov-Krasovskii functional, which are suitable for analysis of every system within the class under consideration. This functional is based on the 
Lyapunov function constructed for the corresponding homogeneous system without delay, and it is assumed that the system without delay is asymptotically stable.

The work plan is as follows. In Section 2, we justify the need to take into account the delay in the management of ships. In Section 3, we have provided the necessary definitions and statements for further understanding. In Section 4, we present a construction of a complete type functional for a scalar homogeneous equation and prove a theorem on asymptotic stability and instability of the zero solution. We present a general construction of the functional in Section 5.

\section{MARINE TRANSPORTATION AND DELAY PROBLEM}

Assume that we have given mathematical model of the ship motion with a constant speed under sea wave action [12]

$$
\begin{aligned}
& \dot{\beta}=a_{11} \cdot \beta+a_{12} \cdot \omega+b_{1} \cdot \delta+h_{1} \cdot d(t), \\
& \dot{\omega}=a_{21} \cdot \beta+a_{22} \cdot \omega+b_{2} \cdot \delta+h_{2} \cdot d(t), \\
& \dot{\phi}=\omega, \\
& \dot{\delta}=u, y=\phi .
\end{aligned}
$$

Here $\beta$ is a drift angle, $\omega$ is an angular velocity, $\phi$ is a yaw angle, and $\delta$ is a rudder deflection.

Let us consider a transport vessel with a displacement of about 4000 ton, moving at a given course at a constant speed of $15 \mathrm{~m} / \mathrm{s}$. If you enter variables $\beta=x_{1}, \omega=x_{2}, \phi=x_{3}$, the problem of steady motion is reduced to the stabilization of the zero solution of the system

$$
\begin{aligned}
& \dot{x}_{1}(t)=-0.140 \cdot x_{1}(t)-0.634 \cdot x_{2}(t)-0.0285 \cdot \delta(t), \\
& \dot{x}_{2}(t)=-0.108 \cdot x_{1}(t)-1.08 \cdot x_{2}(t)+0.0360 \cdot \delta(t), \\
& \dot{x}_{3}(t)=x_{2}(t) \\
& \dot{\delta}(t)=u(t) .
\end{aligned}
$$

We assume that the coefficients of the basic control law $u=\boldsymbol{k}_{x}\left(\boldsymbol{x}-\boldsymbol{x}_{0}\right)+k_{0} \delta, \boldsymbol{x}_{0}=$ ( $\left.\begin{array}{lll}0 & 0 & \phi_{0}\end{array}\right)$, are found, and this control provides the desired quality of the plant dynamics. Here

$$
\begin{aligned}
& \boldsymbol{k}_{x}=\left(\begin{array}{ll}
k_{1} & k_{2} \\
k_{1} & =1.44, k_{2}
\end{array}\right), \\
& k_{2}=-5.28, k_{3}=-3.16, k_{0}=-0.680 .
\end{aligned}
$$

In Fig. 1 the function graphs of $\phi(t)$ and $\delta(t)$ for a closed system in the absence of an external perturbation are shown. Here the movement is generated by the command signal $\phi_{0}=10^{\circ}$ supplied to the control system through the control $u(t)=\boldsymbol{k}_{x}\left(\boldsymbol{x}(t)-\boldsymbol{x}_{0}\right)+$ $k_{0} \delta(t)+\xi$.
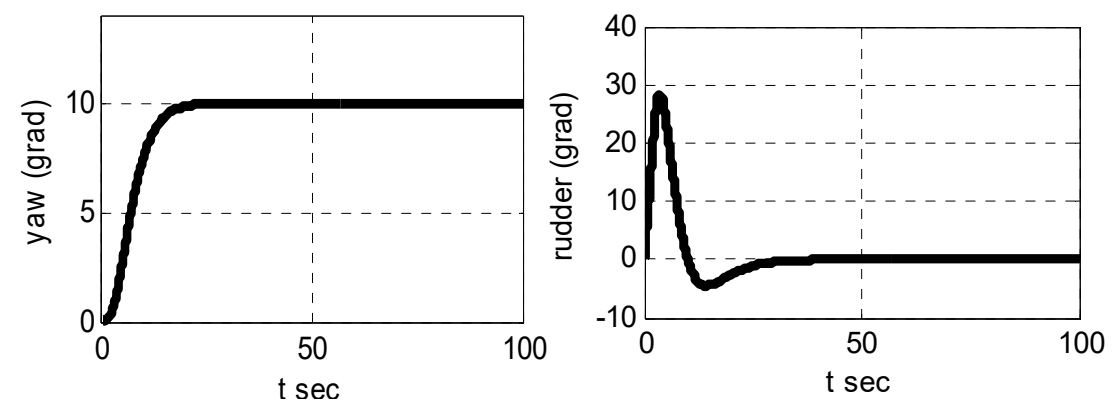

Figure 1: Graphs of functions $\phi(t)$ and $\delta(t)$ respectively for the reference closed system. 
Now we introduce the delay $h=3 c$ into the feedback $u(t-h)$. Fig. 2 shows similar graphs for the $\mathrm{u}$ control object

$$
\begin{aligned}
& \dot{\boldsymbol{x}}(t)=\boldsymbol{A} \boldsymbol{x}(t)+\boldsymbol{b} \delta(t), \\
& \dot{\delta}(t)=u(t-h) .
\end{aligned}
$$
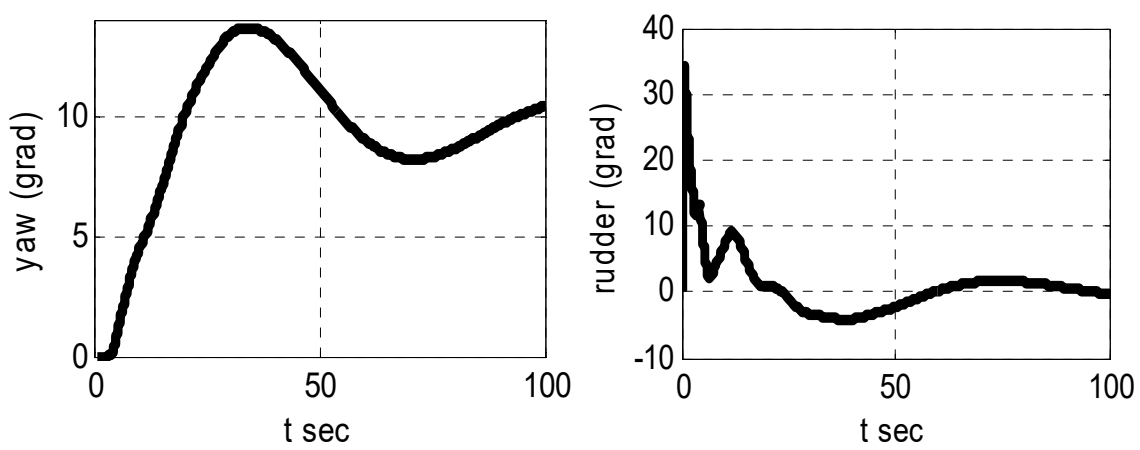

Figure 2: Graphs of functions $\phi(t)$ and $\delta(t)$, respectively, for the closed-loop system with delay $h=3 c$.

Comparison of the processes in Figs 1 and 2 show that the presence of a delay significantly worsens the qualitative characteristics of the vessel's movement in this mode.

Note that increasing the delay to $h=6 c$ causes a loss of stability, as illustrated in Fig. 3.
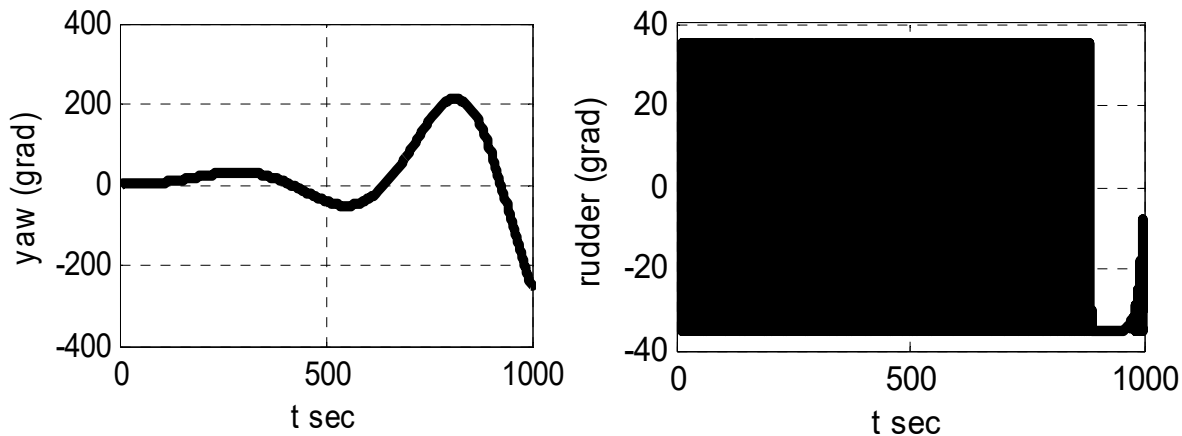

Figure 3: Graphs of functions $\phi(t)$ and $\delta(t)$, respectively, for the closed-loop system with delay $h=6 c$.

Thus, it can be concluded that the neglect of delay in the synthesis of feedback leads to a significant deterioration in the dynamic quality. Therefore, it is necessary to investigate the effect of delay in the control law on the dynamics of the system.

For the design of an autopilot, in [12], the linear control law $u=\boldsymbol{k}_{x}\left(\boldsymbol{x}-\boldsymbol{x}_{0}\right)+k_{0} \delta$ was used. However, it is worth mentioning that in some application the use of nonlinear control laws is required [13]. Moreover, the impact of delay in the control scheme on the dynamics of a system should be taken into account [1], [12], [13]. In the work [2] the nonlinear control 
law was considered as a homogeneous function of the order of a larger unit, and the closed system was described by a system of difference-differential equations of the lagging type. In this paper we consider homogeneous systems of difference-differential equations of neutral type.

Consider the system of equations

\section{PRELIMINARY}

$$
\dot{x}(t)+D \cdot \dot{x}(t-\tau)=f(x(t))+g(x(t-h)), \quad t \geq t_{0} .
$$

Here $x(t) \in R^{n}, \tau>0$ and $h>0 \mathrm{~h}>0$ are constant delays, and all eigenvalues of the matrix $D$ modulo less than one. We also assume that vector functions $f(x), g(y)$ are positively homogeneous of order $\mu>1 \mu>1$, i.e. $f(c x)=c^{\mu} f(x), g(c y)=c^{\mu} g(y)$ for all $x, y \in R^{n}, c \in R^{+}$, and these functions are twice continuously differentiable. Since the system eqn (1) is stationary, we take $t_{0}=0$ and denote by $x(t, \phi)$ the solution of the system eqn (1) corresponding to the initial function $\phi(t)$.

Next, we consider the initial function $\phi(t)$ to be continuous, piecewise continuously differentiable and satisfying the conditions $\|\phi\|_{h} \leq H$ and $\|\dot{\phi}\|_{\tau} \leq H$. If $v \in(-\tau, 0]$ is the point of the discontinuity derivative, then we assume the equality of $\dot{\phi}(v)=\dot{\phi}(v+0)$.

Definition 1. The solution of the system eqn (1) is a vector function $x(t, \phi)$ defined for $t \geq 0$, continuous and piecewise continuously differentiable. In the future, we will use the symbol $x(t)$ or $x_{t}$ to solve $x(t, \phi)$.

Remark 2. It is obvious that the solution $x(t, \phi)$ is a continuous and piecewise continuously differentiable function, and at the break points $t \in\{v+m \cdot \tau, \quad m=1,2, \ldots\}$ of the derived solution the equality $\dot{x}(t, \phi)=\dot{x}(t+0, \phi)$ is true.

Lemma 3. Let function $\psi(t, \tau)$ be continuous in $t$, continuously differentiable in $t$ and piecewise continuous in $\tau$.

Then the function $I(t)=\int_{t-h}^{t} \psi(t, v) d v$ is continuous and piecewise continuously differentiable, and the equations

$$
\begin{gathered}
\dot{I}(t+0)=\psi(t, t+0)-\psi(t, t-h+0)+\int_{t-h}^{t} \frac{\partial \psi(t, v)}{\partial t} d v \\
\dot{I}(t-0)=\psi(t, t-0)-\psi(t, t-h-0)+\int_{t-h}^{t} \frac{\partial \psi(t, v)}{\partial t} d v
\end{gathered}
$$

are valid.

Corollary 4. If $x(t)$ is the solution of eqn (1), then the following equations are valid for $t \geq \max \{\tau, h\}$ :

$$
\begin{gathered}
x(t-h)=x(t)-\int_{t-h}^{t} \dot{x}(v) d v \\
g(x(t-h))=g(x(t))-\int_{t-h}^{t}\left(\frac{\partial g(x(v))}{\partial x(v)}\right)^{T} \cdot \dot{x}(v) d v \\
\frac{d}{d t}\left(\int_{t-\tau}^{t}(\alpha+\beta \cdot(v-t)) \cdot\|\dot{x}(v)\|^{2} d v\right) \\
=\alpha \cdot\|\dot{x}(t)\|^{2}-(\alpha-\beta \cdot \tau) \cdot\|\dot{x}(t-\tau)\|^{2}-\beta \cdot \int_{t-\tau}^{t} \dot{x}^{2}(v) d v .
\end{gathered}
$$


The equalities eqns (2)-(4) are also true at points $t \in\{\tau+m \cdot h, \quad m=1,2, \ldots\}$ of the break of function $\dot{x}(t)$.

Along with the eqn (1) system, we consider a system of ordinary differential equations without delay

$$
(E+D) \cdot \dot{z}(t)=f(z(t))+g(z(t)) .
$$

Since the functions $f(x)$ and $g(y)$ are positive-homogeneous, then the inequality $\|f(x)\| \leq M \cdot\|x\|^{\mu}$ is true, in which $M=\max \left\{\|f(x)\|,\|x\|=1, x \in R^{n}\right\}$, and the inequality $\|g(y)\| \leq M_{1} \cdot\|y\|^{\mu}$ is true, in which $M_{1}=\max \left\{\|g(y)\|,\|y\|=1, y \in R^{n}\right\}$.

Since $\frac{\partial g(y)}{\partial y_{j}}, j=1, \ldots, n$ is positive-homogeneous functions of order $\mu-1$, there exists such a constant $M_{2}$ that $\left\|\frac{\partial g(y)}{\partial y}\right\| \leq M_{2} \cdot\|y\|^{\mu-1}$.

The following stability results form the basis for our further constructions in this report.

Theorem 5. Let $w(z)$ be a differentiable positive homogeneous function satisfying the conditions $a_{1} \cdot\|z\|^{\beta} \leq w(z) \leq a_{2} \cdot\|z\|^{\beta}$ and $\beta>\mu+1$.

If system (3) is asymptotically stable, then there are exists a positive homogeneous function $V(z)$ such that the following holds:

a) $V(z)$ is a twice differentiable positive homogeneous function;

b) the function $V(z)$ is differentiable along the solution of system eqn (5), and

$$
\frac{d V(z(t))}{d t}=\left(\frac{\partial V(z)}{\partial z}\right)^{T} \cdot(E+D)^{-1} \cdot(f(z)+g(z))=-w(z) .
$$

Consider the equation

\section{EXAMPLE: SCALAR EQUATION}

$$
\dot{x}(t)+d \cdot \dot{x}(t-h)=a \cdot x^{3}(t)+b \cdot x^{3}(t-h), \quad t \geq 0,
$$

in which $|d|<1$ and $a+b<0$.

Corollary 4 for eqn (6) takes the form:

Corollary 6. If $x(t)$ is the solution of eqn (6), then the following equations are valid for $t \geq h$ :

$$
\begin{gathered}
x(t-h)=x(t)-\int_{t-h}^{t} \dot{x}(v) d v \\
x^{3}(t-h)=x^{3}(t)-3 \cdot \int_{t-h}^{t} x^{2}(v) \cdot \dot{x}(v) d v \\
\frac{d}{d t}\left(\int_{t-h}^{t}(\alpha+\beta \cdot(v-t)) \cdot \dot{x}^{2}(v) d v\right) \\
=\alpha \cdot \dot{x}^{2}(t)-(\alpha-\beta \cdot h) \cdot \dot{x}^{2}(t-h)-\beta \cdot \int_{t-h}^{t} \dot{x}^{2}(v) d v .
\end{gathered}
$$

The equalities eqns (7)-(9) are also true at points $t \in\{\tau+m \cdot h, \quad m=1,2, \ldots\}$ of the break of function $\dot{x}(t)$.

We are going to construct a complete type functional, positively defined in some neighborhood of zero, and which solve problem of the asymptotic stability of the zero solution of eqn (6).

To this end, we consider several auxiliary functionals and their derivatives along the solutions of this equation.

Let 


$$
v_{1}\left(x_{t}\right)=(x(t)+d \cdot x(t-h))^{2},
$$

then

$$
\begin{gathered}
\left.\frac{d v_{1}\left(x_{t}\right)}{d t}\right|_{(4)}=2 \cdot(x(t)+d \cdot x(t-h)) \cdot\left(a \cdot x^{3}(t)+b \cdot x^{3}(t-h)\right) \\
=2 \cdot(a+b) \cdot(1+d) \cdot x^{4}(t)-6 \cdot b \cdot(1+d) \cdot x(t) \cdot \int_{t-h}^{t} x^{2}(v) \cdot \dot{x}(v) d v \\
-2 \cdot d \cdot\left(a \cdot x^{3}(t)+b \cdot x^{3}(t-h)\right) \cdot \int_{t-h}^{t} \dot{x}(v) d v .
\end{gathered}
$$

Using obvious inequalities

$$
2 \cdot\left|x(t) \cdot \int_{t-h}^{t} x^{2}(v) \cdot \dot{x}(v) d v\right| \leq \frac{1}{\varepsilon} \cdot x^{2}(t) \cdot \int_{t-h}^{t} x^{4}(v) d v+\varepsilon \cdot \int_{t-h}^{t} \dot{x}^{2}(v) d v
$$

and

$$
\begin{array}{r}
2 \cdot\left|\left(a \cdot x^{3}(t)+b \cdot x^{3}(t-h)\right) \cdot \int_{t-h}^{t} \dot{x}(v) d v\right| \\
\leq \frac{1}{\varepsilon} \cdot h \cdot\left(a \cdot x^{3}(t)+b \cdot x^{3}(t-h)\right)^{2}+\varepsilon \cdot \int_{t-h}^{t} \dot{x}^{2}(v) d v,
\end{array}
$$

we obtain an estimate

$$
\begin{aligned}
& \left.\frac{d v_{1}\left(x_{t}\right)}{d t}\right|_{(4)} \leq 2 \cdot(a+b) \cdot(1+d) \cdot x^{4}(t)+\varepsilon \cdot(3 \cdot|b| \cdot(1+d)+|d|) \cdot \int_{t-h}^{t} \dot{x}^{2}(v) d v \\
& +\frac{1}{\varepsilon} \cdot\left[3 \cdot|b| \cdot(1+d) \cdot x^{2}(t) \cdot \int_{t-h}^{t} x^{4}(v) d v+h \cdot|d|\left(a \cdot x^{3}(t)+b \cdot x^{3}(t-h)\right)^{2}\right] .
\end{aligned}
$$

Define

$$
v_{2}\left(x_{t}\right)=x^{4}(t)
$$

then

$$
\begin{aligned}
& \left.\frac{d v_{2}\left(x_{t}\right)}{d t}\right|_{(4)}=4 \cdot x^{3}(t) \cdot\left(-d \cdot \dot{x}(t-h)+\left(a \cdot x^{3}(t)+b \cdot x^{3}(t-h)\right)\right) \\
= & 4 \cdot(a+b) \cdot x^{6}(t)+4 \cdot x^{3}(t) \cdot\left(-d \cdot \dot{x}(t-h)-3 b \cdot \int_{t-h}^{t} x^{2}(v) \cdot \dot{x}(v) d v\right) .
\end{aligned}
$$

Therefore

$$
\begin{aligned}
\left.\frac{d v_{2}\left(x_{t}\right)}{d t}\right|_{(4)} \leq 4 & \cdot(a+b) \cdot x^{6}(t)+2 \cdot \tilde{\varepsilon} \cdot\left(3 \cdot|b| \cdot \int_{t-h}^{t} \dot{x}^{2}(v) d v+|d| \cdot \dot{x}^{2}(t-h)\right) \\
& +\frac{2}{\tilde{\varepsilon}} \cdot\left[3 \cdot|b| \cdot x^{6}(t) \cdot \int_{t-h}^{t} x^{4}(v) d v+|d| \cdot x^{6}(t)\right] .
\end{aligned}
$$

Let

$$
v_{3}\left(x_{t}\right)=\int_{t-h}^{t}\left(2 h+\left(1-d^{2}\right) \cdot(v-t)\right) \cdot \dot{x}^{2}(v) d v
$$

then 


$$
\begin{aligned}
& \left.\frac{d v_{3}\left(x_{t}\right)}{d t}\right|_{(4)}=2 h \cdot \dot{x}^{2}(t)-h \cdot\left(1+d^{2}\right) \cdot \dot{x}^{2}(t-h)-\left(1-d^{2}\right) \cdot \int_{t-h}^{t} \dot{x}^{2}(v) d v \\
& =-h \cdot\left(1-d^{2}\right) \cdot \dot{x}^{2}(t-h)-4 h \cdot d \cdot \dot{x}(t-h) \cdot\left(a \cdot x^{3}(t)+b \cdot x^{3}(t-h)\right) \\
& \quad+2 h \cdot\left(a \cdot x^{3}(t)+b \cdot x^{3}(t-h)\right)^{2}-\left(1-d^{2}\right) \cdot \int_{t-h}^{t} \dot{x}^{2}(v) d v .
\end{aligned}
$$

Hence

$$
\begin{gathered}
\left.\quad \frac{d v_{3}\left(x_{t}\right)}{d t}\right|_{(4)} \leq-h \cdot\left(1-d^{2}\right) \cdot \dot{x}^{2}(t-h)+2 \cdot \hat{\varepsilon} \cdot h \cdot|d| \cdot \dot{x}^{2}(t-h) \\
+2 \cdot h \cdot\left(1+\frac{|d|}{\hat{\varepsilon}}\right) \cdot\left(a \cdot x^{3}(t)+b \cdot x^{3}(t-h)\right)^{2}-\left(1-d^{2}\right) \cdot \int_{t-h}^{t} \dot{x}^{2}(v) d v .
\end{gathered}
$$

If

$$
v_{4}\left(x_{t}\right)=\int_{-h}^{0}(2 h+v) \cdot x^{4}(t+v) d v
$$

then

$$
\left.\frac{d v_{4}\left(x_{t}\right)}{d t}\right|_{(4)}=2 h \cdot x^{4}(t)-h \cdot x^{4}(t-h)-\int_{-h}^{0} x^{4}(t+v) d v .
$$

Take some positive numbers $A, B, C$, and consider the functional

$$
v\left(x_{t}\right)=v_{1}\left(x_{t}\right)+A \cdot v_{2}\left(x_{t}\right)+B \cdot v_{3}\left(x_{t}\right)+C \cdot v_{4}\left(x_{t}\right) \text {. }
$$

The functional $v\left(x_{t}\right)$ is obviously positive definite and has an infinitesimal upper limit.

We calculate the derivative of this functional along the solutions of eqn (6). Using the above estimates of eqns (10)-(13), and applying the inequality

$$
\left(a \cdot x^{3}(t)+b \cdot x^{3}(t-h)\right)^{2} \leq\left(a^{2}+b^{2}\right) \cdot\left(x^{6}(t)+x^{6}(t-h)\right),
$$

we obtain the estimate

$$
\left.\frac{d v\left(x_{t}\right)}{d t}\right|_{(4)} \leq I_{1}+I_{2}+I_{3}+I_{4}+I_{5}
$$

in which

$$
\begin{gathered}
I_{1}=x^{4}(t) \cdot\left[2 \cdot(a+b) \cdot(1+d)+4 \cdot A \cdot(a+b) \cdot x^{2}(t)+2 \cdot C \cdot h\right. \\
+\left(\frac{h}{\varepsilon} \cdot|d| \cdot\left(a^{2}+b^{2}\right)\right) \cdot x^{2}(t)+\frac{2 \cdot A}{\tilde{\varepsilon}}\left(|d|+3 \cdot|b| \cdot \int_{t-h}^{t} x^{4}(v) d v\right) \cdot x^{2}(t) \\
\left.+2 \cdot B \cdot h \cdot\left(1+\frac{|d|}{\hat{\varepsilon}}\right) \cdot\left(a^{2}+b^{2}\right) \cdot x^{2}(t)\right], \\
I_{2}=x^{4}(t-h) \cdot\left[-h \cdot C+\frac{h \cdot|d| \cdot\left(a^{2}+b^{2}\right)}{\varepsilon} \cdot x^{2}(t-h)\right. \\
\left.\left.+2 \cdot B \cdot h \cdot\left(1+\frac{|d|}{\hat{\varepsilon}}\right) \cdot\left(a^{2}+b^{2}\right) \cdot x^{2}(t-h)\right)\right], \\
I_{3}=\int_{t-h}^{t} x^{4}(v) d v \cdot\left[-C+\frac{3 \cdot|b| \cdot(1+d)}{\varepsilon} \cdot H^{2}\right], \\
I_{4}=\dot{x}^{2}(t-h) \cdot\left[-B \cdot h \cdot\left(1-d^{2}\right)+2 \cdot B \cdot \hat{\varepsilon} \cdot h \cdot|d|+2 \cdot A \cdot \tilde{\varepsilon} \cdot|d|\right],
\end{gathered}
$$




$$
I_{5}=\int_{t-h}^{t} \dot{x}^{2}(v) d v \cdot\left[-B \cdot\left(1-d^{2}\right)+\varepsilon \cdot(3 \cdot|b| \cdot(1+d)+|d|)+6 \cdot A \cdot \tilde{\varepsilon} \cdot|b|\right] .
$$

Set the values of the coefficients $A=B=1, \quad C \cdot h=-(a+b) \cdot(1+d) / 4$ of the functional $v\left(x_{t}\right)$, and the parameters

$$
\varepsilon=\tilde{\varepsilon}=\hat{\varepsilon}=\frac{1-d^{2}}{2} \cdot \min \left\{\frac{1}{3 \cdot|b| \cdot(3+d)+|d|} ; \quad \frac{h}{2 \cdot(1+h) \cdot|d|}\right\} .
$$

Then we have the inequality

$$
I_{4} \leq-\frac{h \cdot\left(1-d^{2}\right)}{2} \cdot \dot{x}^{2}(t-h)
$$

and

$$
I_{5} \leq-\frac{\left(1-d^{2}\right)}{2} \cdot \int_{t-h}^{t} \dot{x}^{2}(v) d v .
$$

Next, we define parameter $H$ from condition

$$
H^{2}=\min \left\{1 ; \quad \frac{C \cdot \varepsilon}{6|b| \cdot(1+d)} ; \quad \frac{C \cdot \varepsilon}{\left(a^{2}+b^{2}\right)(4 \varepsilon+6|d|)} ; \frac{2 \cdot C \cdot h \cdot \varepsilon}{2|d|+h \cdot\left\{6|b|+\left(a^{2}+b^{2}\right)(2 \varepsilon+3|d|)\right\}}\right\},
$$

so inequalities $I_{3} \leq-\frac{C}{2} \cdot \int_{t-h}^{t} x^{4}(v) d v, I_{2} \leq-\frac{C \cdot h}{2} \cdot x^{4}(t-h)$ and $I_{1} \leq(a+b) \cdot(1+d)$. $x^{4}(t)$ hold true.

Since the functional $v\left(x_{t}\right)$ satisfies the conditions of Krasovsky's theorem, the next statement is true.

Theorem 7. If $|d|<1$ and $a+b<0$, then the zero solution of eqn (6) is asymptotically Lyapunov stable.

Theorem 8. If $|d|<1$ and $a+b>0$, then the zero solution of eqn (6) is Lyapunov unstable.

Proof. To be carried out in the same way, if you select the following Lyapunov functional

$$
v\left(x_{t}\right)=-v_{1}\left(x_{t}\right)-A \cdot v_{2}\left(x_{t}\right)+B \cdot v_{3}\left(x_{t}\right)+C \cdot v_{4}\left(x_{t}\right) \text {. }
$$

At first, we study a system (1)

\section{BASIC RESULTS}

$$
\dot{x}(t)+D \cdot \dot{x}(t-h)=f(x(t))+g(x(t-h)),
$$

in which $\tau=h$.

Theorem 9. If system eqn (3) is asymptotically stable and $\|D\|=d<1$, then the trivial solution of system eqn (14) is also asymptotically stable.

Proof. To analyze the asymptotic stability of the zero solution of the system of eqn (14) we will construct the functional of the complete type that satisfy Krasovsky's theorem.

Let the functions $V(z)$ and $w(z)$ satisfy the conditions of theorem 5, with the condition $6 \mu>3 \beta>4 \mu-2$.

We consider several auxiliary functionals and their derivatives along the solutions of this system.

Let

$$
v_{1}\left(x_{t}\right)=V(z)
$$

in which

$$
z=(E+D)^{-1} \cdot(x(t)+D \cdot x(t-h))
$$


Then

$$
\left.\frac{d v_{1}\left(x_{t}\right)}{d t}\right|_{(10)}=\left(\frac{\partial V(z)}{\partial z}\right)^{T} \cdot(E+D)^{-1} \cdot(f(x(t))+g(x(t-h)) .
$$

Using equalities eqns (2), (3) and equality

$$
\left\|\frac{\partial V(z)}{\partial z}-\frac{\partial V(x(t))}{\partial(x(t))}\right\|=\left\|\frac{\partial V^{2}\left[x(t)-\sigma \cdot(E+D)^{-1} \cdot D \cdot \int_{t-h}^{t} \dot{x}(v) d v\right]}{\partial z^{2}} \cdot(E+D)^{-1} \cdot D \cdot \int_{t-h}^{t} \dot{x}(v) d v\right\|,
$$

in which $\sigma \in(0,1)$, we obtain an estimate

$$
\begin{aligned}
& \left.\frac{d v_{1}\left(x_{t}\right)}{d t}\right|_{(10)} \leq-w(x(t))+\varepsilon \cdot b_{1} \cdot \int_{t-h}^{t}\|\dot{x}(v)\|^{2} d v \\
+ & \frac{c_{1} \cdot H^{\beta-2}}{\varepsilon} \cdot\left(\|x(t)\|^{\beta}+\|x(t-h)\|^{\beta}+\int_{t-h}^{t}\|x(v)\|^{\beta} d v\right) .
\end{aligned}
$$

Define

$$
v_{2}\left(x_{t}\right)=(V(x(t)))^{2} .
$$

Then

$$
\begin{gathered}
\left.\frac{d v_{2}\left(x_{t}\right)}{d t}\right|_{(10)}=2 \cdot V(x(t)) \cdot\left(\frac{\partial V(x(t))}{\partial(x(t))}\right)^{T} \\
\left.\times\left(-D \cdot \dot{x}(t-h)+f(x(t))+g(x(t))-\int_{t-h}^{t}\left(\frac{\partial g(x(v))}{\partial x(v)}\right)^{T} \cdot \dot{x}(v) d v\right)\right) .
\end{gathered}
$$

Therefore

$$
\begin{aligned}
\left.\frac{d v_{2}\left(x_{t}\right)}{d t}\right|_{(10)} \leq-2 & \left.\cdot V(x(t)) \cdot w(x(t))+\tilde{\varepsilon} \cdot b_{2} \cdot\left(\|\dot{x}(t-h)\|^{2}+\int_{t-h}^{t}\|\dot{x}(v)\|^{2} d v\right)\right) \\
& +\frac{c_{2} \cdot H^{3 \beta-4 \mu+2}}{\tilde{\varepsilon}} \cdot\left(\|x(t)\|^{\beta}+\int_{t-h}^{t}\|x(v)\|^{\beta} d v\right) .
\end{aligned}
$$

Let

$$
v_{3}\left(x_{t}\right)=\int_{t-h}^{t}\left(2 h+\left(1-d^{2}\right) \cdot(v-t)\right) \cdot\|\dot{x}(v)\|^{2} d v
$$

then

$$
\begin{gathered}
\left.\frac{d v_{3}\left(x_{t}\right)}{d t}\right|_{(10)}=2 h \cdot\|\dot{x}(t)\|^{2}-h \cdot\left(1+d^{2}\right) \cdot\|\dot{x}(t-h)\|^{2}-\left(1-d^{2}\right) \cdot \int_{t-h}^{t}\|\dot{x}(v)\|^{2} d v \\
=2 h \cdot\|-D \cdot \dot{x}(t-h)+f(x(t))+g(x(t-h))\|^{2} \\
-h \cdot\left(1+d^{2}\right) \cdot\|\dot{x}(t-h)\|^{2}-\left(1-d^{2}\right) \cdot \int_{t-h}^{t}\|\dot{x}(v)\|^{2} d v .
\end{gathered}
$$

Evaluating the resulting expression in the same way as a similar expression in the proof of Theorem 7, and taking into account the equality of $\|D\|=d<1$, we obtain an estimate of

$$
\left.\frac{d v_{3}\left(x_{t}\right)}{d t}\right|_{(10)} \leq-h \cdot\left(1-d^{2}\right) \cdot\|\dot{x}(t-h)\|^{2}+2 \cdot \hat{\varepsilon} \cdot h \cdot d \cdot\|\dot{x}(t-h)\|^{2}
$$




$$
+2 \cdot h \cdot\left(1+\frac{d}{\hat{\varepsilon}}\right) \cdot\|f(x(t))+g(x(t-h))\|^{2}-\left(1-d^{2}\right) \cdot \int_{t-h}^{t}\|\dot{x}(v)\| d v .
$$

If

$$
v_{4}\left(x_{t}\right)=\int_{-h}^{0}(2 h+v) \cdot\|\dot{x}(t+v)\|^{\beta} d v,
$$

then

$$
\left.\frac{d v_{4}\left(x_{t}\right)}{d t}\right|_{(10)}=2 h \cdot\|\dot{x}(t)\|^{\beta}-h \cdot\|\dot{x}(t-h)\|^{\beta}-\int_{-h}^{0}\|\dot{x}(t+v)\|^{\beta} d v .
$$

The proof of this theorem is completed in the same way as theorem 7 .

Remark 10. An obvious modification of the proof allows us to verify the validity of this theorem for the original system eqn (1).

Remark 11. Under the conditions of Theorem 9, the zero solution of the system eqn (14) is asymptotically stable at any delays of $\tau>0$ and $h \geq 0$.

Theorem 12. If system eqn (5) is asymptotically stable and the modules of all eigenvalues of the matrix $D$ are less than one, then the trivial solution of the system eqn (1) is asymptotically stable.

Proof. If all eigenvalues of matrix $D$ are modulo less than one, then there is a similarity transformation $\widetilde{D}=S^{-1} D S$ such that $\|\widetilde{D}\|<1$. Therefore, the transformation of variables $x=S y$ leads the system eqn (1) to a new system

$$
\dot{y}(t)+\widetilde{D} \cdot \dot{y}(t-\tau)=S^{-1} f(S y(t))+S^{-1} g(S y(t-h)),
$$

for which the conditions of Theorem 9 are satisfied.

\section{CONCLUSION}

In this paper we propose a method for constructing of Lyapunov-Krasovsky functionals for homogeneous difference-differential systems of neutral type. These functionals allows us to estimate the permissible limits of errors of the ship's control system parameters, the limits of permissible oscillations of dynamic characteristics and so on. In addition, the constructed functionals can serve as the basis for the optimal damping controller. The developed theory and proposed algorithms should improve the safety of sailing and the safety of maneuvering of sea vessels in heavy conditions of navigation.

\section{REFERENCES}

[1] Veremey, E.I., Dynamical correction of control laws for marine ship's accurate steering. Journal of Marine Science and Application, 13, pp. 127-133, 2014.

[2] Aleksandrov, A.Y., Aleksandrova, E.B. \& Zhabko, A.P., Stability analysis of some classes of nonlinear switched systems with time delay. International Journal of Systems Science, 48(10), pp. 2111-2119, 2017.

[3] Veremey, E.I., Linear Feedback Systems, Lan: St. Petersburg, Moscow, Krasnodar, pp. 230-247, 2013. (In Russian.)

[4] Kharitonov, V.L., An extension of the prediction scheme to the case of systems with both input and state delay. Automatica, 50(1), pp. 211-217, 2014.

[5] Zubov, V.I., Methods of A.M. Lyapunov and Their Applications, P. Noordhoff Ltd., Groningen, The Netherlands, pp. 104-129, 1964.

[6] Aleksandrov, A.Y. \& Zhabko, A.P., On the asymptotic stability of solutions of nonlinear systems with delay. Siberian Math. Journal, 53(3), pp. 393-403, 2012. 
[7] Aleksandrov, A.Y., Hu, G.-D. \& Zhabko, A.P., Delay-independent stability conditions for some classes of nonlinear systems. IEEE Trans. Autom. Control, 59(8), pp. 22092214, 2014.

[8] Aleksandrov, A.Y. \& Zhabko, A.P., Delay-independent stability of homogeneous Systems. Applied Mathematics Letters, 34, pp. 43-50, 2014.

[9] Alexandrova, I.V. \& Zhabko, A.P., At the junction of Lyapunov-Krasovskii and Razumikhin approaches. IFAC Papers OnLine, 51(14), pp. 147-152, 2018.

[10] Kharitonov, V.L. \& Zhabko, A.P., Lyapunov-Krasovskii approach to the robust stability analysis of time-delay systems. Automatica, 39, pp. 15-20, 2003.

[11] Medvedeva, I.V. \& Zhabko, A.P., Synthesis of Razumikhin and Lyapunov-Krasovskii approaches to stability analysis of time-delay systems. Automatica, 51, pp. 372-377, 2015.

[12] Veremey, T.I., Dynamical correction of control laws for marine ships' accurate steering. Journal of Marine Science and Application, 13(2), pp. 127-133, 2014. DOI: $10.1007 / \mathrm{s} 11804-014-1250-1$.

[13] Fossen, T.I., Guidance and Control of Ocean Vehicles, Wiley: New York, 1994. 